\title{
Resuscitation after cardiac surgery awareness: an Egyptian national survey
}

\author{
Moslem Abdelghafar ${ }^{1 *}$ (D), Taher Abdelmoneim², Alaa Mohamed ${ }^{3}$ and Mohamed Abdalla ${ }^{4}$
}

\begin{abstract}
Background: Cardiac surgery patients have different resuscitative needs than other patients who experience inhospital cardiac arrest; this was addressed in the guidelines. However, it is unknown how widely the guidelines are practiced, or a training protocol is followed in different cardiac surgery units in Egypt.

Methods: A 21-question survey was created and included: Participant demographics, prevalence of cardiac arrest, cardiac arrest protocol, emergency resternotomy technique, training protocols. Survey was disseminated through social media messaging platforms during the period between November 2020 and January 2021.

Results: Ninety-five responses were from 11 centres across Egypt. In total, $68.5 \%$ of the respondents were surgeons, $76.8 \%$ of participants were junior surgeons. For patients who go into VF after cardiac surgery, respondents would attempt a median of 3 shocks with only $24.2 \%$ commencing defibrillation shocks before external cardiac massage, whilst the majority initiating CPR immediately and performing emergency resternotomy in a median time of 10 min. In total, 56.8\% would give $1 \mathrm{mg}$ of adrenaline as soon as the cardiac arrest was established. If a surgeon was not available, only $36.8 \%$ of respondents would allow any trained personnel to perform the emergency resternotomy. Only $9.5 \%$ practice regularly on emergency sternotomies. Seventy-five percent think tailored training is important and staff should be oriented about it in the future.
\end{abstract}

Conclusion: An action plan is required to improve the training of the junior surgeons regarding the Cardiac Advanced Life Support Protocol to implement it in a timely organised manner. This should be endorsed and audited by a national society or body by keeping a national registry and mandatory recertification.

Keywords: Cardiac surgery, Resuscitation, Training, Education

\section{Background}

Every year, over 250,000 patients have cardiac surgery in some 450 centres in Europe [1] and more than 400,000 patients undergo cardiac surgery in the USA at approximately 1200 medical centres [2-4]. During the past decade, there has been an increasing recognition that cardiac surgery patients have different resuscitative needs than other medical and surgical patients who experience inhospital cardiac arrest. The special resuscitative needs of cardiac surgery patients were addressed in the 2021

\footnotetext{
*Correspondence: Moslem_fathy@hotmail.com

${ }^{1}$ Department of Cardiothoracic Surgery, Wythenshawe Hospital,

Manchester, UK

Full list of author information is available at the end of the article
}

European Resuscitation Council (ERC) Guidelines for Resuscitation in the section reviewing cardiac arrest in special circumstances and the 2020 American Heart Association (AHA) guidelines for cardiopulmonary resuscitation and emergency cardiovascular care in special situations $[5,6]$. However, it is unknown how widely the guidelines are practiced, or a training protocol is followed in different units in Egypt. This national survey aims to identify the views and common practice of Egyptian cardiac surgery teams regarding resuscitation after cardiac surgery. 


\section{Methods and materials}

A 21-question survey was created (Table 1) based on the original survey used by the European Association of Cardiothoracic Surgery (EACTS) guidelines committee [7]. Questions included the following topics: Participant demographics, prevalence of cardiac arrest in the intensive care unit, cardiac arrest with ventricular fibrillation or non-shockable rhythm, emergency resternotomy technique, training and arrest protocols. The survey was modified to collect participants' demographics; other questions remained the same as the original survey. Survey dissemination was targeted to staffing of cardiothoracic departments in various institutes through social media platforms such as mobile messaging applications and emails during the time period between November 2020 and January 2021; this was our preference due to COVID-19 pandemic to allow better reach and in line with social distancing national guidance. Ethics approval and informed consent have been waived by the institutional review board.

\section{Demographics}

The first eight questions were used to obtain demographic data on the expertise of the respondent, the size of the unit and the prevalence of cardiac arrest and emergency resternotomy in the unit where the respondent worked.

\section{Data cleaning}

Prior to analysis, the data from all respondents were analysed independently and excluded if the multiple choice, numerical or text responses indicated that the survey had been incorrectly completed or if multiple respondents came from the same email address. Respondents were excluded if there were no responses to over $50 \%$ of the questions or if the numerical data responses were impossible (i.e. more arrests than operations performed in that unit).

\section{Statistical analysis}

Continuous data are presented as median, mean, standard deviation and range or only as median if the data was significantly skewed using the Kolmogorov-Smirnov test. Categorical data was presented as percentages. Data was presented and analysed using SPSS 13.0 (Statistical Package for the Social Sciences, SPSS Inc., Chicago, USA).

\section{Results}

Of 126 responses, 95 were suitable for inclusion. Thirtyone responses were deleted due to duplication or incorrect completion. We have responses from 11 centres across Egypt, $68.5 \%$ of the respondents were surgeons whilst cardiac anaesthetists and intensivists formed $12.6 \%$ and $18.9 \%$ respectively. The majority of participants were non-consultants/middle-grade doctors comprising $76.8 \%$; consultant participation was $23.2 \%$.
The median number of cases performed by all units was 480 and this ranged from 10 to 3000 annually. The average percentage of cardiac arrests in these units was $7 \%$, and the average percentage of emergency resternotomy after cardiac arrest was $2.4 \%$. Respondents reported that the median survival to hospital discharge of all arrests was $33 \%$.

In patients who arrest with VF or VT, only $24.2 \%$ of respondents would commence 1-3 defibrillation shocks and then perform external cardiac massage (ECM), with the majority initiating CPR immediately (Fig. 1).

Regarding the sequence of defibrillation attempts interspersed with ECM, 50.5\% of respondents would perform three attempts at defibrillation without intervening ECM. In total, $49.5 \%$ would perform single defibrillation attempts interspersed with ECM at 1- or 2-min intervals. In total, $56.8 \%$ of respondents would give $1 \mathrm{mg}$ of adrenaline as soon as the cardiac arrest was established. Only $6.3 \%$ of respondents thought that it should be given rarely or not at all (Fig. 2).

For patients who go into VF in less than $24 \mathrm{~h}$ after cardiac surgery, respondents would attempt a mean of 3 defibrillation shocks and would perform emergency resternotomy in a median time of $10 \mathrm{~min}$ and in $15 \mathrm{~min}$ if the rhythm was not VF. Whilst for patients who suffer cardiac arrest more than $24 \mathrm{~h}$ after the surgery results were, a median of 5.6 attempts of defibrillation and a median of $17.6 \mathrm{~min}$ to perform emergency resternotomy (Table 2).

A series of questions were asked on the conduct of an emergency resternotomy in patients who have suffered a cardiac arrest (Table 3). If a surgeon was not immediately available, $36.8 \%$ of respondents would allow a suitably trained non-surgeon to perform the emergency resternotomy whilst $58.9 \%$ of respondents would not accept anyone except a surgeon to perform the resternotomy. Of respondents who would allow a non-surgeon to perform an emergency resternotomy, 33.7\% would allow anyone trained for the procedure to do it, $14.4 \%$ would allow an anaesthesiologist, $17.9 \%$ would allow intensive care doctor and $12.6 \%$ would allow a junior grade doctor to perform an emergency resternotomy. Only $9.5 \%$ and $3.2 \%$ would allow a theatre scrub nurse and a senior intensive care nurse respectively to do this.

A total of $49.4 \%$ of the participants have never practised any local training to perform an emergency sternotomy; however, $34.7 \%$ believe it is a must-have. Forty-one percent of the respondents state they occasionally practice or talk with the staff about guidance in the event of a cardiac arrest. Only $9.5 \%$ practice regularly on emergency sternotomies.

Twenty-five percent assume that current training is sufficient and does not need modification or additional patient tailoring, whilst $75 \%$ think tailored training is important and staff should be encouraged to undertake it in the future. 
Table 1 Depicting survey questions

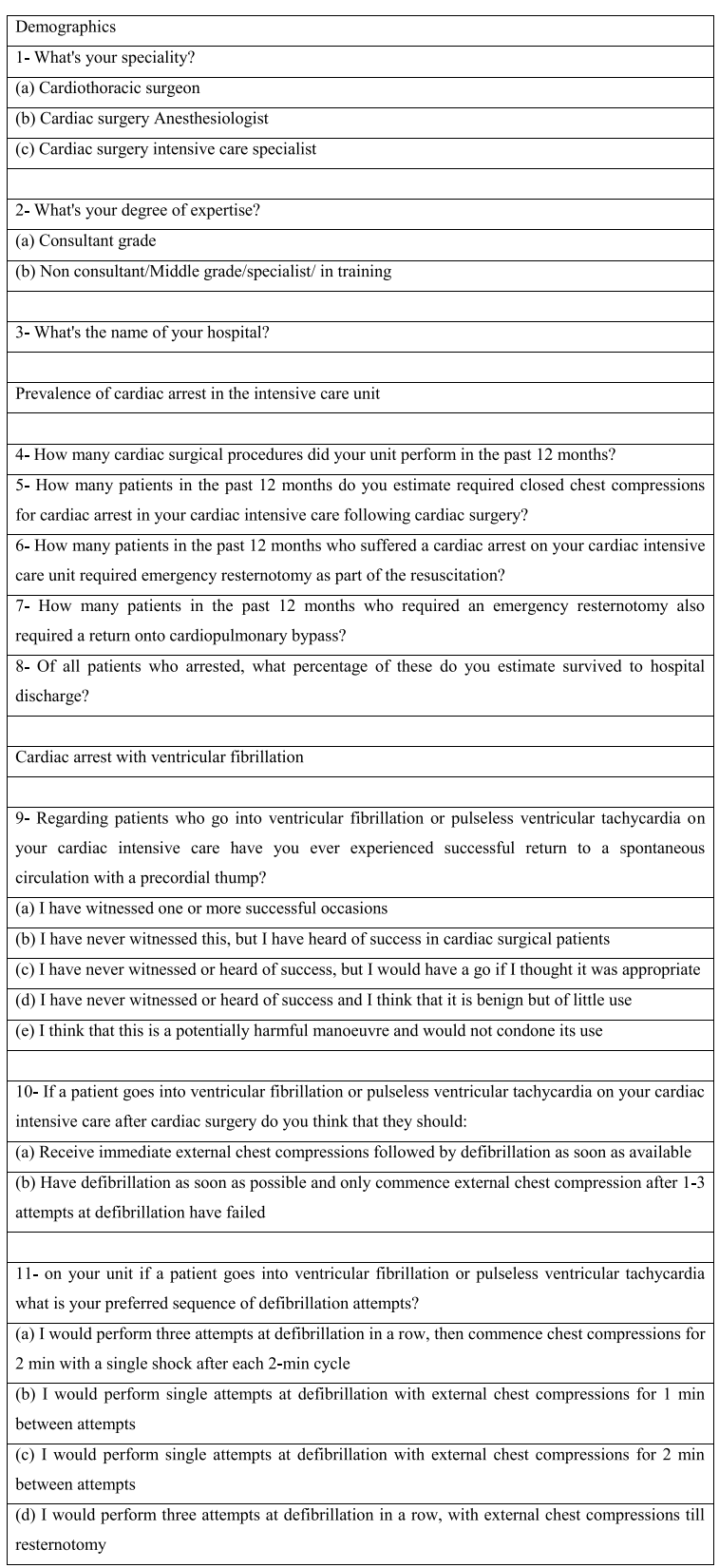

Table 1 (continued)

12- For patients in established cardiac arrest when do you think that administration of epinephrine (adrenaline) is warranted?

(a) As soon as possible

(b) after 2 min of external massage

(c) After 3-5 min of external massage

(d) After 5-10 min of external massage

(e) Only after continued cardiac arrest despite emergency resternotomy (1) Only in exceptional circumstances and should not be part of routine cardiac arrest management after cardiac surgery

13- If you had to name a number of defibrillation attempts or a rough time at which you felt that a patient less than $24 \mathrm{~h}$ after cardiac surgery in ventricular fibrillation should have emergency resternotomy what would it be? (number of attempts and number of minutes)

14- If you had to name a number of defibrillation attempts or a rough time at which you felt that a patient MORE than $24 \mathrm{~h}$ after cardiac surgery in ventricular fibrillation should have emergency resternotomy what would it be? (number of attempts and number of minutes) Cardiac arrest where the rhythm is not VF or pulseless VT.

15- With regard to patients who arrest after cardiac surgery, but the rhythm is not ventricular fibrillation or ventricular tachycardia. In how many minutes should emergency resternotomy be performed if the patient is WITHIN $24 \mathrm{~h}$ of surgery?

16- With regard to patients who arrest after cardiac surgery, but the rhythm is not ventricular fibrillation or ventricular tachycardia. When should emergency resternotomy be performed if the patient is MORE THAN $24 \mathrm{~h}$ of surgery? Emergency resternotomy technique

17- With regard to emergency chest re-opening

(a) A surgeon should always perform this and non-surgeons should never do this

(b) If no surgeon is available rapidly then you would be happy for a non-surgeon to perform this if trained to do it

(c) if no surgeon is available rapidly then you would be happy for a non-surgeon to perform this even if they have had no formal training

18- In the exceptional circumstance where no surgeon is immediately available to perform the emergency resternotomy in an arrest, who would you be happy to do this instead? (multiple responses allowed)

(a) An anaesthesiologist

(b) A surgeon's assistant

(c) A theatre nurse

(d) A junior doctor

(e) A senior intensive care nurse

(f) Nobody except surgeons should do this Training and arrest protocols

19- In your unit do you practice and provide training in the process of emergency chest re-opening with your staff in the intensive care?

(a) We never practice, and this is not necessary

(b) We never practice this, but this might be a good idea

(c) We talk about this informally to our staff and they have experience of this

(d) We have occasionally practiced this

(e) We regularly practice this

20- With regard to our current guidelines for resuscitation for patients who arrest in the cardiac intensive care unit.

(a) I advocate current resuscitation guidelines as proposed by the European Resuscitation Council or the American Heart Association on our unit

(b) I do not agree with current resuscitation guidelines and we have our own agreed protocol on our unit

(c) I do not agree with current resuscitation guidelines. I would act as I see fit, although we do not have our own agreed protocol on the unit.

(d) I have not actually read either of the European resuscitation council or EACTS updated guidelines on resuscitation

21- With regard to staff training for cardiac arrest after cardiac surgery in the intensive care. (a) Current training available is adequate despite it not being tailored to patients after cardiac surgery and no further training is needed in our unit

(b) We give additional training tailored to patients after cardiac surgery and no additional training is needed

(c) Training tailored to patients after cardiac surgery may be useful for our staff in the future

(d) Training tailored to patients after cardiac surgery is important and should be given in the future 


\section{0- RESPONSES IF A PATIENT GOES INTO VF OR VT}

- Receive immediate external chest compressions followed by defibrillation

-1-3 defibrillations followed by external chest compressions

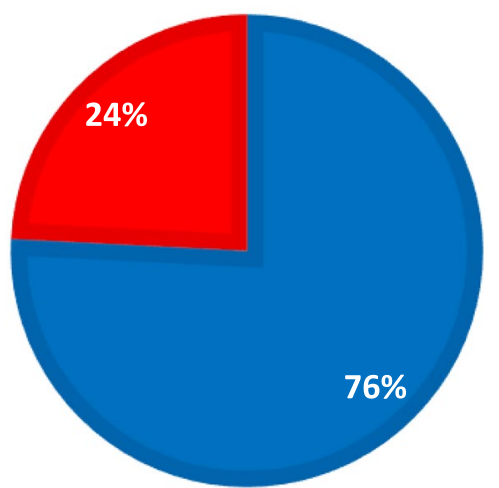

Fig. 1 Percentage of respondents commencing ECM or DC shocks in a VF arrest situation

\section{2- RESPONSES FOR ADRENALINE ADMINSTRATION IN ESTABLISHED CARDIAC ARREST}
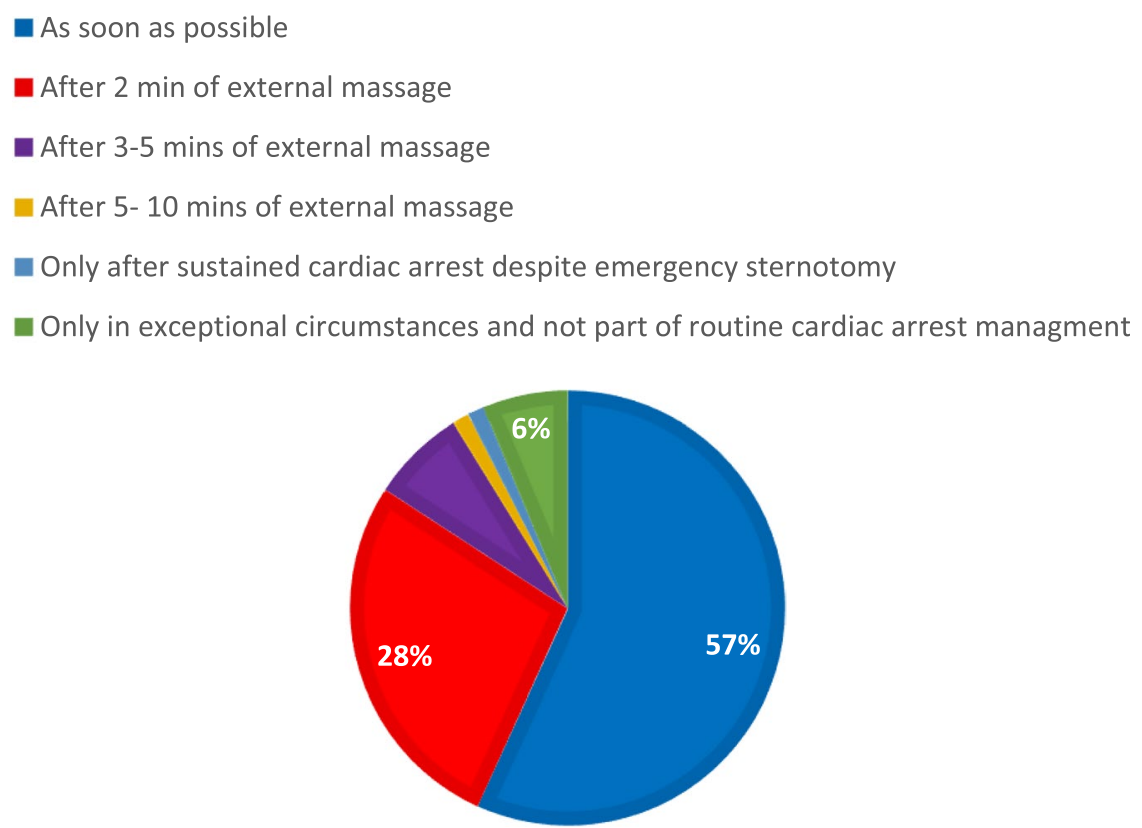

Fig. 2 Adrenaline administration patterns after cardiac arrest

In total, $70.5 \%$ of all respondents advocate the current guidelines for resuscitation published by the ERC, EACTS and the AHA for use on their patients; however,
$5 \%$ disagree with the guidelines and have their own local protocol. Meanwhile, $24.2 \%$ have not read the guidelines. 
Table 2 Median of defibrillation attempts and time to perform emergency resternotomy

\begin{tabular}{|c|c|c|c|}
\hline & $\begin{array}{l}\text { Number of } \\
\text { responses }\end{array}$ & Median & Range \\
\hline \multicolumn{4}{|c|}{ Number of defibrillation attempts before resternotomy in VFNT } \\
\hline Cardiac arrest $<24 \mathrm{~h}$ & 95 & 3 shocks & $14(\min 1, \max 15)$ \\
\hline Cardiac arrest $>24 \mathrm{~h}$ & 95 & 5 shocks & $19(\min 1, \max 20)$ \\
\hline \multicolumn{4}{|c|}{ Time to resternotomy where initial rhythm is VFNT } \\
\hline Cardiac arrest $<24 \mathrm{~h}$ & 95 & $10 \mathrm{~min}$ & $29(\min 1, \max 30)$ \\
\hline Cardiac arrest $>24 \mathrm{~h}$ & 95 & $17.6 \min$ & $58(\min 2, \max 60)$ \\
\hline \multicolumn{4}{|c|}{ Time to resternotomy where initial rhythm is asystole/PEA } \\
\hline Cardiac arrest $<24 \mathrm{~h}$ & 95 & $15 \min$ & $44(\min 1, \max 45)$ \\
\hline Cardiac arrest $>24 \mathrm{~h}$ & 95 & $17.6 \mathrm{~min}$ & $44(\min 1, \max 45)$ \\
\hline
\end{tabular}

\section{Discussion}

The incidence of cardiac arrest after cardiac surgery is around 0.7-7\% [8-16], EACTS, ERC and AHA endorsed resuscitation guidelines for this special group. Our survey gives an insight into current practices and adoption of guidelines of the Egyptian cardiac surgery centres. To our knowledge, there were no studies on a national level to address post cardiac surgery resuscitation practices.

In our study, $68.5 \%$ of the respondents were cardiac surgeons, of which $76.8 \%$ middle grade/resident/junior doctors. This is representative of first responders to cardiac arrest call in a routine practice thus it is crucial to identify knowledge and practices to evaluate the quality and safety of patient care. However, we found no significant differences in practices amongst respondents from various institutions, consultant and non-consultants, surgeons and non-surgeons.

Our respondents will act in a VT/VF cardiac arrest situation as follows, $75 \%$ will start CPR, $57 \%$ will give adrenaline immediately. All of which are more in line with Advanced Life Support (ALS) or Advanced Cardiac Life Support (ACLS) protocols and not the protocol dedicated for cardiac surgery.

The current guidelines advocate, once cardiac arrest is identified, to assess the rhythm first and not to commence chest compressions, reason being the possibility of presence of shockable rhythm such as VF or pulseless VT in $25-50 \%$ of cases. If a shockable rhythm is identified, chest compressions could be delayed for up to 1 min to deliver 3 shocks as this might spare the traumatic chest compressions to a fresh sternotomy wound and avoid complications of cardiac/graft injury $[17,18]$.

In the cardiac surgical patient, the efficacy of defibrillation reduces by $10 \%$ for every minute delay, in addition, success rates for immediate sequential shocks for VF or pVT decline from $78 \%$ with the first shock to $14 \%$ with the third, therefore, immediate defibrillation with three sequential attempts at 150 Joules is advised [19]. Whilst in severe bradycardia or asystole, it is advisable before starting chest compressions to turn the pacing to emergency setting or DDD mode, 90 beats, maximum amplitude.

No study concluded benefit or harm of administering adrenaline during resuscitation of the postoperative cardiac surgical patient; however, the risk of administering adrenaline in conventional doses is with profound hypertension, bleeding or tearing of vessel anastomoses on return of spontaneous circulation (ROSC), which can precipitate catastrophic harm or further cardiac arrest [20]. Therefore, the recommendation to administer adrenaline is to be delayed until reversible causes of arrest are excluded and directed by a senior clinician experienced in their use. Adrenaline remains a useful drug in peri-arrest situations in smaller doses.

Of concern, almost $60 \%$ would not prefer anyone but the surgeon to perform a resternotomy, we believe the main culprit is medicolegal claims and pursuits. Nonetheless, $17.9 \%$ would allow intensive care doctors trained for emergency sternotomy to perform it in case of cardiac arrest. This stems from the fact that junior surgeons in many Egyptian cardiac units are allocated to manage cardiac intensive care, thus, being familiar with surgical problems and have enough skills to perform an emergency sternotomy.

In total, $70.5 \%$ of respondents advocate the current guidelines for resuscitation yet only $10 \%$ train regularly. Practicing protocol-based arrest management has been shown to reduce by $50 \%$ the time to chest reopening, reduce complications resulting from the resternotomy after cardiac surgery and improve survival [21-25]. Thus, the need to raise the awareness and training of the junior surgical doctors and intensive care staff with current guidance and emergency sternotomy protocols is paramount.

A structured national registry and regular auditing are key features to achieve the compliance, training, and monitoring of trainees; in addition, regular mandatory recertification is crucial to maintain an up-to-date knowledge of the current pool of surgeons and fellows. This could be endorsed by the cardiothoracic society body or national health service in the country.

The core message for our trainees and fellows; external chest compressions are ineffective in tamponade, extreme hypovolemia due to bleeding. Brain damage will occur in $5 \mathrm{~min}$; the only way to save those patients is to perform a rapid smooth emergency resternotomy.

\section{Limitations}

Our study has several limitations. Survey respondents are almost always self-selected, not everyone who receives a survey is likely to answer it despite offered incentives, which explains the small number of respondents. However, this was the best available alternative for multi-centre data collection due to COVID19 pandemic and the national guidance for social distancing. 
Table 3 A series of questions on the conduct of an emergency resternotomy in patients who suffered a cardiac arrest

\begin{tabular}{|c|c|c|}
\hline & Responses & percentages \\
\hline (6) Precordial thump (total) & 95 & \\
\hline (a) Witnessed & 36 & $37.9 \%$ \\
\hline (b) Heard of success & 15 & $15.8 \%$ \\
\hline (c) Have a go & 14 & $14.7 \%$ \\
\hline (d) Of little use & 18 & $18.9 \%$ \\
\hline (e) Potentially harmful & 12 & $12.6 \%$ \\
\hline (7) Defibrillation or ECM for VF & 95 & \\
\hline (a) Immediate ECM & 72 & $75.8 \%$ \\
\hline (b) Immediate defibrillation & 23 & $24.2 \%$ \\
\hline (8) Sequence of shocks for VF & 95 & \\
\hline (a) Three attempts, 2 min ECM then single shocks & 34 & $35.8 \%$ \\
\hline (b) Single attempts with $1 \mathrm{~min} E C M$ & 12 & $12.6 \%$ \\
\hline (c) Single attempts with 2 min ECM & 35 & $36.8 \%$ \\
\hline (d) Three attempts, ECM till resternotomy & 14 & $14.7 \%$ \\
\hline (9) When is adrenaline warranted & 95 & \\
\hline (a) As soon as possible & 54 & $56.8 \%$ \\
\hline (b) After 2 min of ECM & 26 & $27.4 \%$ \\
\hline (c) After 3-5 min after ECM & 8 & $8.4 \%$ \\
\hline (d) Only after emergency resternotomy & 1 & $1.1 \%$ \\
\hline (e) Only in exceptional circumstances & 6 & $6.3 \%$ \\
\hline (15) Emergency resternotomy & 95 & \\
\hline (a) A surgeon should always do this & 56 & $58.9 \%$ \\
\hline (b) A trained non-surgeon could do this & 35 & $36.8 \%$ \\
\hline (c) Any non-surgeons could do this & 4 & $4.2 \%$ \\
\hline (17) Do you train for emergency resternotomy & 95 & \\
\hline (a) We never practise, not necessary & 14 & $14.7 \%$ \\
\hline (b) We never practise might be good idea & 33 & $34.7 \%$ \\
\hline (c) Informal talks and experience & 11 & $11.6 \%$ \\
\hline (d) We have occasionally practised & 28 & $29.5 \%$ \\
\hline (e) We regularly practise & 9 & $9.5 \%$ \\
\hline (18) Current guidelines for the ICU & 95 & \\
\hline (a) I advocate the ERC/AHA 2005 guidelines & 67 & $70.5 \%$ \\
\hline (b) I do not agree with these, we have our own protocol & 1 & $1.1 \%$ \\
\hline (c) I do not agree with these, we have no protocol & 4 & $4.2 \%$ \\
\hline (d) I have not read the ERC/AHA guidelines & 23 & $24.2 \%$ \\
\hline (19) Current training & 95 & \\
\hline (a) It is adequate currently but not tailored & 17 & $17.9 \%$ \\
\hline (b) We give additional training & 7 & $7.4 \%$ \\
\hline (c) Tailored training might be useful & 18 & $18.9 \%$ \\
\hline (d) Tailored training is important and should be given & 53 & $55.8 \%$ \\
\hline
\end{tabular}

Data regarding the number of procedures, closed chest compressions, resternotomy and going back on bypass rates may not represent the actual figures and numbers as $77 \%$ of the respondents are middle-grade doctors and might not be able to access these institutional numbers readily. Moreover, COVID-19 pandemic has significantly impacted the number of procedures performed and could be a valid reason for the heterogeneity of data from respondents from the same centre.

\section{Conclusions}

This is the first study on a national level to address post-cardiac surgery resuscitation, it revealed a gap in the knowledge and current practices. An action plan is required to train the junior surgeons on the Cardiac Advanced Life Support Protocol, which should be endorsed and audited by a national society or body through a national registry and mandatory recertification. 

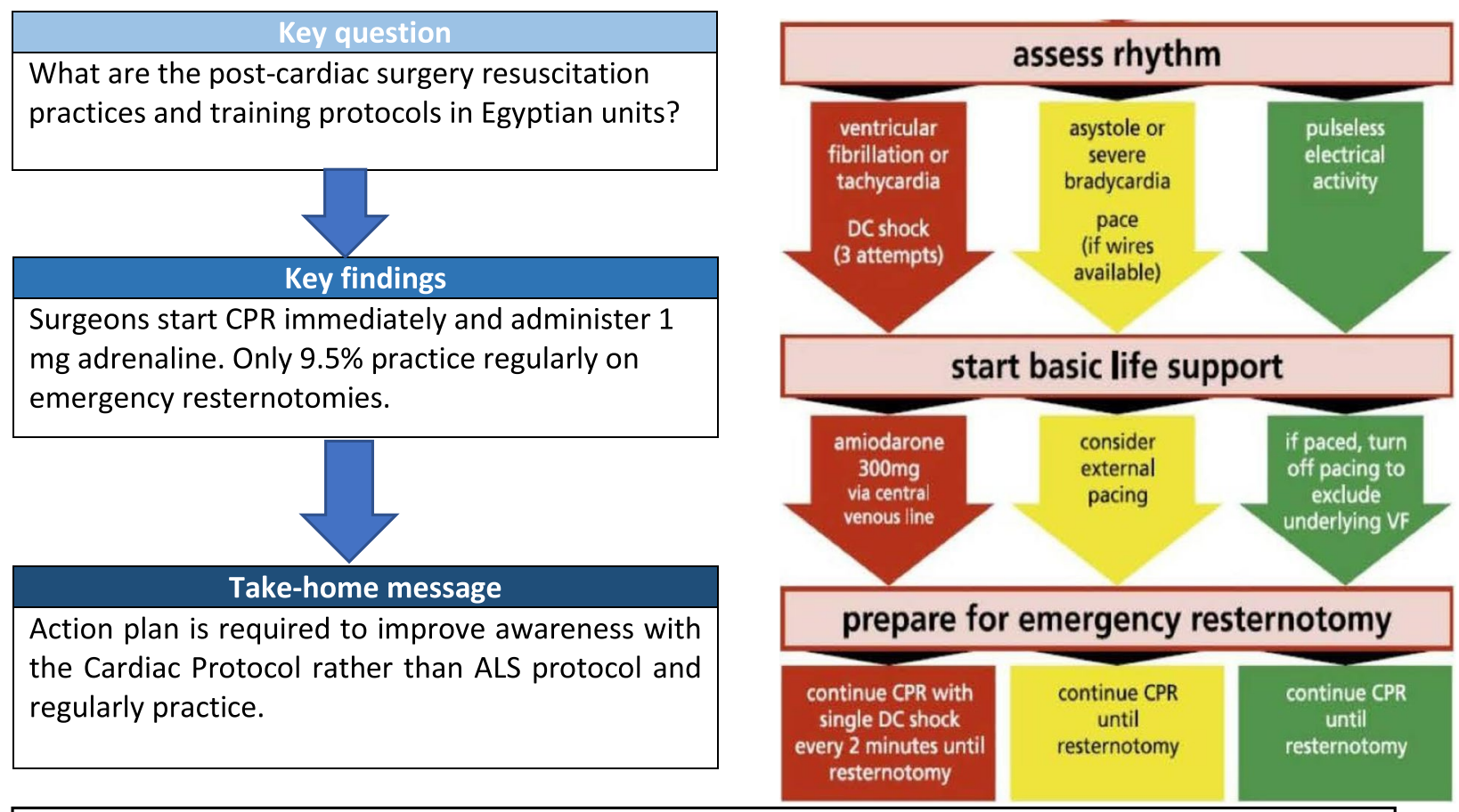

Visual abstract highlighting key findings and post cardiac surgery arrest protocol as per EACTS/STS guidelines.

\section{Abbreviations}

ACLS: Advanced Cardiac Life Support; AHA: American Heart Association; ALS: Advanced Life Support; CPR: Cardiopulmonary resuscitation; EACTS: European Association of Cardiothoracic Surgery; ECM: External cardiac massage; ERC: European resuscitation council; ICU: Intensive care unit; ROSC: Return of spontaneous circulation; PEA: Pulseless electrical activity; STS: Society of Thoracic Surgeons; VF: Ventricular fibrillation;VT: Pulseless ventricular tachycardia.

\section{Acknowledgements}

The abstract has been published in the European Heart Journal supplements, Volume 23, Issue Supplement_D, October 2021, suab069.013 as a winning abstract in CardioAlex 2.1 conference.

\section{Authors' contributions}

Moslem Abdelghafar contributed to conception, design, analysis, interpretation and writing of the work. Taher Abdelmoniem, Alaa Mohamed and Mohamed Abdalla contributed to data acquisition and revising the manuscript. All authors have read and approved the manuscript.

\section{Funding}

The authors received no financial support for the research, authorship and/or publication of this article.

\section{Availability of data and materials}

The data sets used and/or analysed during the current study are available from the corresponding author upon reasonable request.

\section{Declarations}

Ethics approval and consent to participate Not applicable

\section{Consent for publication}

Not applicable

\section{Competing interests}

No competing financial interests exist.

\section{Author details}

${ }^{1}$ Department of Cardiothoracic Surgery, Wythenshawe Hospital, Manchester, UK. ${ }^{2}$ Department of Cardiac Surgery, National Heart Institute, Giza, Egypt. ${ }^{3}$ Department of Cardiothoracic Surgery, El-Hussine Hospital, Al-Azhar University, Cairo, Egypt. ${ }^{4}$ Department of Cardiac Surgery, Shebein El Kom Teaching Hospital, Shebein El Kom, Egypt.

Received: 1 September 2021 Accepted: 5 January 2022

Published online: 07 February 2022

\section{References}

1. Unger $F$ (1993) European survey on cardiac interventions: open-heart surgery, percutaneous transluminal coronary angioplasty and cardiac catheterization in 1993. Cardiovasc Surg 3(6):569-571

2. Jacobs JP, Edwards FH, Shahian DM et al (2010) Successful linking of the Society of Thoracic Surgeons adult cardiac surgery database to Centers for Medicare and Medicaid Services Medicare data. Ann Thorac Surg 90:1150-1157

3. Roger VL, Go AS, Lloyd-Jones DM et al (2012) Heart disease and stroke statistics - 2012 update: a report from the American Heart Association. Circulation 125:e2-e220

4. Girotra S, Lu X, Popescu I, Vaughan-Sarrazin M, Horwitz PA, Cram P (2010) The impact of hospital cardiac specialization on outcomes after coronary 
artery bypass graft surgery: analysis of Medicare claims data. Circ Cardiovasc Qual Outcomes 3:607-614

5. Lott C, Truhlár A, Alfonzo A et al (2021) European resuscitation council guidelines 2021: cardiac arrest in special circumstances. Resuscitation. 161:152-219. https://doi.org/10.1016/j.resuscitation.2021.02.011

6. Panchal AR, Bartos JA, Cabañas JG et al (2020) Part 3: adult basic and advanced life support: 2020 American Heart Association guidelines for cardiopulmonary resuscitation and emergency. Cardiovasc Care 142. https://doi.org/10.1161/CIR.0000000000000916

7. Pottle A, Bullock I, Thomas J, Scott L (2002) Survival to discharge following open chest cardiac compression (OCCC). A 4-year retrospective audit in a cardiothoracic specialist Centre-Royal Brompton and Harefield NHS trust, United Kingdom. Resuscitation 52(3):269-272

8. Adam Z, Adam S, Everngam RL et al (2009) Resuscitation after cardiac surgery: results of an international survey. Eur J Cardiothorac Surg 36(1):29-34

9. Mackay JH, Powell SJ, Osgathorp J, Rozario CJ (2002) Six-year prospective audit of chest reopening after cardiac arrest. Eur J Cardiothorac Surg 22(3):421-425

10. Birdi I, Chaudhuri N, Lenthall K, Reddy S, Nashef SA (2000) Emergency reinstitution of cardiopulmonary bypass following cardiac surgery: outcome justifies the cost. Eur J Cardiothorac Surg 17(6):743-746

11. El-Banayosy A, Brehm C, Kizner L, Hartmann D, Kortke H, Korner MM, Minami K, Reichelt W, Korfer R (1998) Cardiopulmonary resuscitation after cardiac surgery: a two-year study. J Cardiothorac Vasc Anesth 12(4):390-392

12. Anthi A, Tzelepis GE, Alivizatos P, Michalis A, Palatianos GM, Geroulanos S (1998) Unexpected cardiac arrest after cardiac surgery: incidence, predisposing causes, and outcome of open chest cardiopulmonary resuscitation. Chest 113(1):15-19

13. Charalambous CP, Zipitis CS, Keenan DJ (2006) Chest reexploration in the intensive care unit after cardiac surgery: a safe alternative to returning to the operating theater. Ann Thorac Surg 81(1):191-194

14. Wahba A, GotzW, Birnbaum DE (1997) Outcome of cardiopulmonary resuscitation following open-heart surgery. Scand Cardiovasc J 31(3):147-149

15. Kaiser GC, Naunheim KS, Fiore AC, Harris HH, McBride LR, Pennington DG, Barner HB, Willman VL (1990) Reoperation in the intensive care unit. Ann Thorac Surg 49(6):903-907

16. McKowen RL, Magovern GJ, Liebler GA, Park SB, Burkholder JA, Maher TD (1985) Infectious complications and cost-effectiveness of open resuscitation in the surgical intensive care unit after cardiac surgery. Ann Thorac Surg 40(4):388-392

17. Reddy HG, Shih T, Englesbe MJ et al (2013) Analyzing "failure to rescue": is this an opportunity for outcome improvement in cardiac surgery? Ann Thorac Surg 95:1976-1981

18. Dunning J, Levine A, Ley J et al (2017) The society of thoracic surgeons expert consensus for the resuscitation of patients who arrest after cardiac surgery. Ann Thorac Surg 103:1005e20

19. Richardson L, Dissanayake A, Dunning J (2007) What cardioversion protocol for ventricular fibrillation should be followed for patients who arrest shortly post-cardiac surgery? Interact Cardiovasc Thorac Surg 6:799e805

20. Webb ST (2008) Caution in the administration of adrenaline in cardiac arrest following cardiac surgery. Resuscitation 78:101

21. Maccaroni MF, Watson ND, Gaage DL (2013) Managing cardiac arrest after cardiac surgery: the impact of a five year evolving resternotomy policy and a review of the literature. Analg Resusc Curr Res S1:1-7

22. Dunning J, Nandi J, Ariffin S, Jerstice J, Danitsch D, Levine A (2006) The cardiac surgery advanced life support course (CALS): delivering signifcant improvements in emergency cardiothoracic care. Ann Thorac Surg 81:1767-1772

23. Danitsch D, Levine A, Choudrey S, Dunning J, Ariffin S, Jerstice J (2006) Evaluation of a cardiac surgery advanced life support course. Nurs Times 102:30-32

24. Wilson CT, Fisher ES, Welch HG, Siewers AE, Lucas FL (2007) U.S. trends in CABG hospital volume: the effect of adding cardiac surgery programs. Health Aff (Millwood) 26:162-168

25. Horwitz JR, Nichols A, Nallamothu BK, Sasson C, Iwashyna TJ (2013) Expansion of invasive cardiac services in the United States. Circulation 128:803-810

\section{Publisher's Note}

Springer Nature remains neutral with regard to jurisdictional claims in published maps and institutional affiliations.

\section{Submit your manuscript to a SpringerOpen ${ }^{\circ}$ journal and benefit from:}

- Convenient online submission

- Rigorous peer review

- Open access: articles freely available online

- High visibility within the field

- Retaining the copyright to your article

Submit your next manuscript at $\boldsymbol{\nabla}$ springeropen.com 